\title{
Management of subarachnoid hemorrhage with intracerebral hematoma: clipping and clot evacuation versus coil embolization followed by clot evacuation
}

\author{
Kenneth de los Reyes, Aman Patel, Joshua B Bederson, Jennifer A Frontera
}

Department of Neurosurgery, Mount Sinai School of Medicine, New York, New York, USA

\section{Correspondence to Dr Jennifer A Frontera, Neurosurgery, Mount Sinai School of Medicine, One Gustave Levy Place, Box 1136, New York, NY 10010, USA; jenfrontera@hotmail.com}

Portions of this work were presented in abstract form at the Neurocritical Care Conference, November 2009.

Received 18 November 2011 Revised 9 December 2011 Accepted 10 December 2011 Published Online First 3 January 2012

\begin{abstract}
Background Aneurysmal subarachnoid hemorrhage (SAH) with associated intracerebral hemorrhage (ICH) is often treated with concomitant surgical clipping and ICH evacuation. The aim of this study was to determine if aneurysm coiling followed by ICH evacuation is a viable alternative treatment.

Methods A retrospective review was conducted between July 2000 and March 2009 of patients with aneurysmal SAH plus ICH (>30 ml or with midline shift $>5 \mathrm{~mm}$ ) who underwent aneurysm repair (either coiling or clipping) and craniotomy for ICH evacuation.

Demographic and radiographic criteria, time to aneurysm protection, length of stay (LOS), treatment complications, discharge disposition and 3 month functional outcome were compared between groups. Results 0 f $18 \mathrm{SAH}+\mathrm{ICH}$ patients, 10 underwent aneurysm coiling followed by ICH evacuation and eight underwent clipping with $\mathrm{ICH}$ evacuation. Compared with clipped patients, coiled patients had a lower Glasgow Coma Scale score (median 5.5 vs 7.5), higher ICH score (median 3 vs 2), worse modified Fisher score (median 4 vs 3 ) and higher rate of herniation at presentation (50\% vs $25 \%)$. Median time to aneurysm protection was shorter in coiled patients (299 vs 885 min, $p<0.001$ ). Comparing coiled with clipped patients, rates of death (30\% vs $25 \%$ ), poor outcome (70\% vs 50\%), median ICU LOS (20 vs 22 days), median hospital LOS (27 vs 29 days) and total median direct costs ( $\$ 64537$ vs $\$ 61243$ ) were similar, as were complication rates (all $\mathrm{p}>0.05$ ).
\end{abstract}

Conclusions Coiling followed by ICH evacuation is associated with faster time to aneurysm protection and similar outcome, LOS and cost as clipping and evacuation. This may be a viable alternative to clipping and $\mathrm{ICH}$ evacuation.

\section{INTRODUCTION}

The incidence of intracerebral hematoma (ICH) among cases of aneurysmal subarachnoid hemorrhage (SAH) has been reported to be as high as $42-84 \%$ based on CT scans and autopsy series. ${ }^{1-3}$ Concomitant SAH and $\mathrm{ICH}$ portends a worse prognosis than SAH alone. ICH caused by aneurysm rupture has been associated with 30 day mortality rates as high as $80-100 \%^{2}{ }^{4}$ compared with $33-50 \%$ mortality rates in SAH overall. ${ }^{5} 6$

Large space occupying hematomas have traditionally been evacuated with simultaneous aneurysmal clipping. ${ }^{7}$ In 1983, Wheelock et al reported a $75 \%$ mortality rate when ICH evacuation was done alone but a $29 \%$ mortality when the aneurysm was also clipped. ${ }^{7}$ In 1988, Tapaninaho et al reported that 15 of 31 patients died with evacuation and clipping as opposed to $100 \%$ mortality with conservative treatment. ${ }^{8}$ Based on this literature, combined aneurysm clipping and ICH evacuation has become a mainstay of treatment. Unfortunately, despite aggressive management, inhospital mortality rates remain high at $47-58 \%{ }^{3} 910$ in this patient population. Surgical complications such as intraoperative aneurysm rupture related to pressure changes after hematoma evacuation may, in part, explain high mortality rates.

Alternative management strategies that entail aneurysm obliteration with coiling prior to $\mathrm{ICH}$ evacuation may limit aneurysm rebleed rates, and lead to fewer intraoperative complications and shorter duration of anesthesia. A recent study of 30 patients who underwent aneurysm coiling followed by ICH evacuation found that $61 \%$ had a good recovery (Glasgow Outcome Score (GOS) 4-5) at 18 months. ${ }^{11}$

We hypothesize that coil embolization of a ruptured aneurysm followed by ICH evacuation is as safe and efficacious as clipping and ICH evacuation in terms of clinical outcome, hospital length of stay and cost. Furthermore, the time to aneurysm repair is hypothesized to shorten with coil embolization followed by hematoma evacuation.

\section{METHODS \\ Study population}

A retrospective review of all patients with aneurysmal SAH admitted to the Mount Sinai Medical Center between July 2000 and March 2009 was conducted. Patients with International Classification of Diseases, ninth revision, Clinical Modifications (ICD-9-CM) code 430 were identified in the Mount Sinai Neurosurgery Department physician database. A chart and head CT review was conducted to confirm the diagnosis of aneurysmal $\mathrm{SAH}$ in all subjects. Inclusion criteria for analysis were defined as: aneurysmal $\mathrm{SAH}$, aneurysm repair via either clipping or coiling, presence of an $\mathrm{ICH}$ $\geq 30 \mathrm{ml}$ or ICH with midline shift $\geq 5 \mathrm{~mm}$, and ICH evacuation via craniotomy. Patients who underwent aneurysm coiling followed by ICH evacuation were compared with patients who underwent simultaneous aneurysm clipping and ICH evacuation. Exclusion criteria were: traumatic $\mathrm{SAH}$, SAH secondary to vascular dissection, vasculopathy, arteriovenous malformation or other non-aneurysmal causes, and age $<18$ years. 
The study was approved by the Mount Sinai institutional review board.

\section{Clinical management}

All patients received nimodipine every $4 \mathrm{~h}$ for 21 days and phenytoin for 7 days for seizure prophylaxis. All patients received $0.9 \%$ normal saline at a rate of $1 \mathrm{ml} / \mathrm{kg} / \mathrm{h}$ to maintain euvolemia. Aminocaproic acid was administered to prevent aneurysm rebleeding in patients who were within $72 \mathrm{~h}$ of aneurysm rupture and had no history of stroke, venous thromboembolism, myocardial infarction or hypercoagulable state. Aminocaproic acid was stopped within $1-3 \mathrm{~h}$ of angiography to minimize the risk of catheter induced spasm. Potential complications of aminocaproic acid include clot formation, focal ischemic deficits and vasospasm (either spontaneous or catheter induced). Early studies using tranexamic acid to prevent aneurysm re-rupture detected higher rates of infarct if continued through the vasospasm period. ${ }^{12}$ More recent randomized trials of tranexamic acid limited to the first few days after SAH detected reduced rebleeding rates without significant differences in delayed cerebral ischemia. ${ }^{13} 14$ An external ventricular drain was placed if the CT scan demonstrated acute hydrocephalus and/or the patient was non-command following or worse, or had a deteriorating neurological examination due to hydrocephalus. Mannitol $(20 \%$, $1 \mathrm{~g} / \mathrm{kg})$ or $23 \%$ hypertonic saline $(30 \mathrm{ml})$ was administered if there was suspected clinical or radiographic signs of herniation and/or elevated intracranial pressure (ICP). Continuous hypertonic saline infusion $(2 \%$ or $3 \%$ ) was administered at a rate of $1 \mathrm{mg} / \mathrm{kg} / \mathrm{h}$ and titrated to maintain a serum sodium of $150-155 \mathrm{mEq} / \mathrm{dl}$ or $20 \%$ mannitol $0.5 \mathrm{~g} / \mathrm{kg}$ was administered every $6 \mathrm{~h}$ to maintain a serum osmolality of 300-320 mosmol/1 if ICP was elevated. Serum sodium and/or osmolality were checked every $6 \mathrm{~h}$ to titrate osmotic therapy. Prior to aneurysm repair, systolic blood pressure was maintained below $160 \mathrm{~mm} \mathrm{Hg}$ with nicardipine or labetolol continuous infusion.

\section{Angiographic management}

All patients underwent digital subtraction cerebral angiography under general anesthesia to characterize the aneurysm and assess for potential treatment with primary coil embolization with either Gugliemi detachable coils (Boston Scientific, Massachusetts, USA) or Orbit coils (Codman, Massachusetts, USA). The goal of treatment, whether endovascular or open, was complete aneurysm obliteration. The preferred strategy for treatment of aneurysmal SAH at our institution, including in the setting of $\mathrm{ICH}$, was to coil embolize first, regardless of clinical or radiographic herniation, which was always initially treated with mannitol or hyptertonic saline and other conservative measures. Surgical hematoma evacuation with clipping was performed when aneurysm characteristics did not allow for endovascular protection (ie, wide neck, large aneurysm size) and when there were logistical concerns-namely, when the interventionalist was unavailable or there were anticipated delays in the angiography suite.

\section{Surgical management}

Patients were brought emergently from the angiography suite following coil embolization or diagnostic angiography to the operating room where all patients underwent an extended pterional craniotomy. A large curvilinear scalp flap was created followed by a fronto-temporal or fronto-temporal-parietal bone flap removal. The dura was widely incised in a cruciate fashion. Careful hematoma removal was performed where accessible. Surgical obliteration of the aneurysm, if not coiled, was performed with clipping. Partial lobectomy and/or craniectomy was performed based on intraoperative evaluation of brain edema. When significant brain swelling and edema were encountered, the bone flap was marsupialized in the abdominal wall. At the end of each case the dura was loosely reflected onto the brain surface, and dural substitutes or Gelfoam (Pfizer, New York, USA) was placed over it. An epidural drain was placed followed by galeal and scalp closure.

Postoperatively, all patients were managed in the neurointensive care unit with frequent neurologic examinations and/ or ICP monitoring. All patients who had signs or symptoms of vasospasm were initially treated with hypertensivehypervolemic therapy followed by angioplasty and/or intraarterial vasodilator injection, if required. Patients who tolerated clamping of an external ventricular drain for $>24 \mathrm{~h}$ underwent drain removal; otherwise, a ventriculo-pertioneal shunt was placed. After discharge, patients were followed in an outpatient setting by the primary neurosurgeon and interventionalist.

\section{Clinical and radiographic measures}

Baseline demographic data including age and gender, and admission radiographic data including modified Fisher score, ${ }^{15} 16$ $\mathrm{ICH}$ score, ICH volume (calculated using the $\mathrm{ABC} / 2$ method), ${ }^{17}$ $\mathrm{ICH}$ location, presence of intraventricular hemorrhage, midline shift (measured from the septum pellucidum at the level of the pineal gland) and aneurysm size were recorded. Admission neurological status was evaluated with the Hunt-Hess Scale ${ }^{18}$ and Glasgow Coma Scale. ${ }^{19}$ Clinical and radiographic evidence of uncal herniation was documented. Total anesthesia, angiography and operating room time were assessed using the anesthesia record. The time at presentation was the time the patient was first registered at Mount Sinai Hospital. Transfer times from outside hospitals were not included in these calculations. All angiograms and surgeries occurred at Mount Sinai Hospital. Treatment complications were identified through a chart audit.

\section{Outcome measures}

Discharge disposition was dichotomized as poor (expired, discharged to subacute care facility, chronic care facility, hospice or a skilled nursing facility) or good (discharged home, to an acute rehabilitation center, home with a healthcare aide or to an acute care facility). Three month GOS score was dichotomized as poor (expired or severely disabled: GOS 1-3) or good (GOS 4-5). The Mount Sinai Hospital data management services provided information on hospital and ICU length of stay (LOS) and hospitalization costs categorized as: total direct costs, overhead, ICU, surgical and imaging/angiography costs. All cost data were adjusted for inflation to $\$ 2009$.

\section{Statistical analysis}

Demographic and radiographic characteristics, timing of procedures, clinical outcomes, LOS and cost were compared between patients who underwent aneurysm clipping and ICH evacuation and patients who underwent coiling with ICH evacuation using the Mann Whitney $U$ non-parametric test for continuous nonnormally distributed variables and the Fisher exact test for dichotomous variables. Logistic regression analysis was performed to determine predictors of dichotomized functional outcomes. All analyses were performed using SPSS V.17. Significance was set at $\mathrm{p}<0.05$.

\section{RESULTS}

Between July 2000 and March 2009, 265 aneurysmal SAH patients were admitted to the Mount Sinai Medical Center. Of these SAH patients, 25/265 (9\%) had a concomitant ICH 
based on CT review. Ten patients underwent coil embolization followed by ICH evacuation between 2002 and 2009 and eight patients underwent simultaneous aneurysm clipping and $\mathrm{ICH}$ evacuation between 2002 and 2007. Seven patients underwent aneurysm repair without ICH evacuation. Compared with patients with SAH who underwent ICH evacuation, those who did not undergo ICH evacuation tended to have smaller ICH volume, a lower modified Fisher score and less midline shift.

Table 1 compares the demographic and radiographic characteristics of patients who underwent clipping with ICH evacuation with those who underwent coiling followed by $\mathrm{ICH}$ evacuation. Both groups were similar but the coiled patients tended to have lower median Glasgow Coma Scores, higher modified Fisher scores and higher ICH scores. The coiled group was twice as likely to present with clinical signs of herniation at admission compared with the clipped group. The clipped group was more likely to have middle cerebral artery aneurysm rupture although this difference was not statistically significant.

Procedure times for the clipped and coiled groups are shown in table 2. Coiled patients achieved aneurysm obliteration a median of 586 min faster than the clipped group $(p<0.001)$ and had a trend toward shorter overall anesthesia time (309 vs $433 \mathrm{~min}$, $\mathrm{p}=0.091$ ). The total time from presentation until aneurysm repair and ICH evacuation did not differ significantly between the two groups however.

There was one symptomatic procedural complication, which occurred in the coiled group. A patient received aspirin and heparin to prevent thrombus formation on a coil loop projecting into the parent vessel lumen and subsequently had ICH expansion from $23 \mathrm{ml}$ to $50 \mathrm{ml}$. The patient deteriorated and subsequently expired shortly thereafter. A residual aneurysm neck was identified in one clipped patient and one coiled patient on follow-up digital subtraction angiography. One patient suffered aneurysm rebleed which occurred after coil embolization. None of these procedural complications was significantly more common in the clipped or coiled group.

Table 1 Admission characteristics of patients with subarachnoid hemorrhage and intracerebral hemorrhage who underwent aneurysm clipping and intracerebral hemorrhage evacuation compared with patients who underwent coiling followed by intracerebral hemorrhage evacuation

\begin{tabular}{lccc}
\hline & $\begin{array}{c}\text { Clip+ ICH } \\
\text { evacuation }\end{array}$ & $\begin{array}{c}\text { Coil+ ICH } \\
\text { evacuation }\end{array}$ & p Value \\
\hline Age (years) & $56(36-74)$ & $55(41-66)$ & 0.762 \\
Admission Hunt-Hess grade & $4(3-5)$ & $4(3-5)$ & 0.573 \\
Admission Glasgow Coma Scale & $7.5(3-14)$ & $5.5(3-14)$ & 0.573 \\
Clinical herniation at admission & $2(25)$ & $5(50)$ & 0.366 \\
Aneurysm size (mm) & $6.3(2-9)$ & $7.3(2-12)$ & 0.740 \\
Aneurysm location & & & \\
$\quad$ MCA & $7(88)$ & $6(60)$ & 0.391 \\
ACOMM & 0 & $1(10$ & \\
$\quad$ PCOMM & $1(12)$ & $3(30)$ & \\
Modified Fisher score & $3(1-4)$ & $4(1-4)$ & 0.055 \\
ICH volume (ml) & $41(11-65)$ & $45(36-66)$ & 0.318 \\
ICH score & $2(1-3)$ & $3(2-4)$ & 0.097 \\
ICH location & & & \\
$\quad$ Frontal-temporal-parietal & $3(37.5)$ & $7(70)$ & 0.342 \\
$\quad$ Temporal & $5(62.5)$ & $3(30)$ & \\
Intraventricular hemorrhage & $2(25)$ & $5(50)$ & 0.286 \\
Midline shift preoperatively (mm) & $4.7(0-13)$ & $5.3(0-10.5)$ & 0.955 \\
Midline shift postoperatively (mm) & $3.0(0-6)$ & $3.5(0-8)$ & 0.799 \\
Hemicraniectomy & $3(38)$ & $7(70)$ & 0.342 \\
\hline
\end{tabular}

Values are median (range) or $\mathrm{n}(\%)$.

ACOMM, anterior communicating artery; ICH, intracerebral hemorrhage; MCA, middle cerebral artery; PCOMM, posterior communicating artery.
Table 2 Time to aneurysm repair in the clipping with intracerebral hemorrhage evacuation group compared with the coiling with intracerebral hemorrhage evacuation group

\begin{tabular}{|c|c|c|c|}
\hline & $\begin{array}{l}\text { Clip+ ICH } \\
\text { evacuation }\end{array}$ & $\begin{array}{l}\text { Coil+ ICH } \\
\text { evacuation }\end{array}$ & p Value \\
\hline $\begin{array}{l}\text { Time from presentation* to } \\
\text { angiography (min) }\end{array}$ & $174(99-592)$ & $117(52-512)$ & 0.263 \\
\hline Time from presentation to $\mathrm{OR}(\mathrm{min})$ & $532(317-775)$ & $915(152-3981)$ & 0.315 \\
\hline Total OR time (min) & $348(237-436)$ & $193(120-340)$ & 0.001 \\
\hline Total angiography time (min) & $60(18-139)$ & $161(83-187)$ & 0.001 \\
\hline Total anesthesia time (min) & $433(301-492)$ & $309(203-507)$ & 0.091 \\
\hline $\begin{array}{l}\text { Total time from presentation to } \\
\text { aneurysm protection (min) }\end{array}$ & $885(644-1211)$ & $299(120-683)$ & $<0.001$ \\
\hline $\begin{array}{l}\text { Total time from presentation until } \\
\text { end of } O R(\mathrm{~min})\end{array}$ & $885(584-1211)$ & $1146(272-4166)$ & 0.601 \\
\hline
\end{tabular}

Values are median (range).

*Presentation is the time the patient first registered at our institution.

$\mathrm{ICH}$, intracerebral hemorrhage; $\mathrm{OR}$, operating room.

Symptomatic vasospasm occurred in 5/8 (63\%) clipped patients compared with $7 / 10(70 \%)$ coiled patients $(p=1.00)$. Infarct on CT or MRI due to delayed cerebral ischemia was diagnosed in $3 / 8(38 \%)$ clipped patients and 1/10 (10\%) coiled patients $(p=0.569)$.

Mortality and functional outcome at discharge and 3 months were also similar between the two groups (table 3 ). The type of aneurysm repair procedure (clip with ICH evacuation or coil followed by ICH evacuation) did not predict death, discharge disposition or 3 month functional outcome on univariate logistic regression analysis. Due to the small sample size, we were unable to identify any significant predictors of outcome in this cohort (table 4).

Median ICU LOS was 22 (range 5-41) days in the clipped group compared with 20 (range 3-34) days in the coiled group $(\mathrm{p}=0.573)$ and median hospital LOS was 29 (range 5-63) days in the clipped group versus 27 (range 3-26) days in the coiled group $(p=0.965)$. Likewise, overall direct costs and categorical breakouts of cost did not reveal a significant difference between the two groups (table 5).

\section{DISCUSSION}

Recent SAH management guidelines mention no specific recommendations regarding treatment of $\mathrm{ICH}$ and $\mathrm{SAH}$ other than that open surgery or combined strategies may be used successfully depending on patient selection. ${ }^{20}$ This study demonstrates that aneurysm coiling followed by ICH evacuation may be a viable alternative management strategy compared with concomitant clipping and ICH evacuation in patients with both $\mathrm{SAH}$ and ICH. In our small study, we found that aneurysm coiling prior to clot evacuation led to more rapid aneurysm obliteration, without any significant difference in procedural complications, functional outcome, LOS or cost. With a $3-4 \%$ risk of rebleeding in the first $24 \mathrm{~h}$ after ictus and a $1-2 \%$ risk everyday afterward, securing a ruptured aneurysm in a timely

Table 3 Functional outcomes at discharge and 3 months

\begin{tabular}{llll}
\hline & $\begin{array}{l}\text { Clip + ICH } \\
\text { evacuation (n (\%)) }\end{array}$ & $\begin{array}{l}\text { Coil+ ICH } \\
\text { evacuation (n (\%)) }\end{array}$ & p Value \\
\hline Dead & $2(25)$ & $3(30)$ & 0.617 \\
Poor discharge disposition & $5(63)$ & $6(60)$ & 0.647 \\
Poor 3 month outcome & $4(50)$ & $7(70)$ & 0.630 \\
(GOS 1-3) & & & \\
\hline
\end{tabular}

GOS, Glasgow Coma Scale; ICH, intracerebral hemorrhage. 
Table 4 Univariate predictors of death, poor discharge disposition and poor 3 month outcome $(n=18)$

\begin{tabular}{|c|c|c|c|}
\hline & Dead (OR $(95 \% \mathrm{Cl}), \mathrm{p}$ value) & $\begin{array}{l}\text { Poor discharge disposition } \\
\text { (OR }(95 \% \mathrm{Cl}) \text {, p value) }\end{array}$ & $\begin{array}{l}\text { Poor } 3 \text { month outcome } \\
\text { (OR }(95 \% \mathrm{CI}) \text {, p value) }\end{array}$ \\
\hline Coil + evacuation versus clip + evacuation & $1.3(0.2$ to 10.5$), p=0.814$ & $0.5(0.1$ to 3.8$), p=0.505$ & - \\
\hline Age & $1.1(0.9$ to 1.2$), p=0.303$ & $1.1(1.0$ to 1.3$), p=0.061$ & $1.2(0.9$ to 1.3$), p=0.403$ \\
\hline Admission Glasgow Coma Scale & $1.0(0.8$ to 1.3$), p=0.912$ & $1.1(0.9$ to 1.4$), p=0.395$ & $0.9(0.6$ to 1.4$), p=0.644$ \\
\hline Herniation at admission & $0.8(0.1$ to 10.9$), p=0.852$ & $0.3(0.0$ to 3.0$), p=0.277$ & - \\
\hline Modified Fisher score & $1.5(0.4$ to 5.1$), p=0.529$ & $0.7(0.2$ to 2.3$), p=0.605$ & $0.6(0.1$ to 5.7$), p=0.686$ \\
\hline Preoperative midline shift & $1.1(0.8$ to 1.4$), p=0.652$ & $1.0(0.7$ to 1.3$), p=0.928$ & $1.1(0.5$ to 2.5$), p=0.751$ \\
\hline Total time to aneurysm protection & $1.0(1.0$ to 1.0$), p=0.879$ & $1.0(1.0$ to 1.0$), p=0.139$ & $1.0(1.0$ to 1.0$), p=0.381$ \\
\hline Total time from presentation until end of $\mathrm{OR}$ & $1.0(1.0$ to 1.0$), p=0.456$ & $1.0(1.0$ to 1.0$), p=0.607$ & $1.0(1.0$ to 1.0$), p=0.575$ \\
\hline ICU length of stay & $0.9(0.8$ to 1.1$), p=0.393$ & $0.9(0.9$ to 1.1$), p=0.490$ & $1.1(0.9$ to 1.4$), p=0.269$ \\
\hline Hospital length of stay & $0.9(0.9$ to 1.0$), p=0.124$ & $1.0(0.9$ to 1.0$), p=0.773$ & $1.1(0.9$ to 1.3$), p=0.314$ \\
\hline
\end{tabular}

$\mathrm{ICH}$, intracerebral hemorrhage; $\mathrm{OR}$, operating room.

fashion is of utmost importance. ${ }^{20}$ Additionally, as aneurysm rebleeding is the single most preventable cause of mortality and poor functional outcome after $\mathrm{SAH}^{21}{ }^{22}$ demonstrating a treatment strategy that leads to more rapid aneurysm obliteration without increased risk is meaningful. Rapid aneurysm protection is especially salient in the context of concurrent $\mathrm{ICH}$ as aneurysm re-rupture rates have been reported to be three times as high in the presence of $\mathrm{ICH}$ compared with without $\mathrm{ICH}^{23}$ We found that aneurysms were secured approximately $5 \mathrm{~h}$ from presentation in the coiled group compared with $15 \mathrm{~h}$ in the clipped group. This difference was not due to surgical postponement in the clipping cohort. In fact, the clipped group arrived in the operating room in a median of $523 \mathrm{~min}(8.7 \mathrm{~h})$ from presentation compared with $915 \mathrm{~min}(15.25 \mathrm{~h})$ for the coiled group. The longer time to secure the aneurysm in the clipped group was due to the time incurred obtaining an initial angiogram, followed by transport and operating room set-up. This time might be shortened in facilities that have combined angiography and operating room suites. Due to our small sample size we were unable to demonstrate a significant association between time to aneurysm obliteration and outcome. Others, however, have shown improved outcomes with faster aneurysm treatment. In a series of 50 patients with $\mathrm{SAH}$ and $\mathrm{ICH}, 80 \%$ of whom were treated with aneurysm clipping and ICH evacuation while $2 \%$ underwent coiling and ICH evacuation, aneurysm obliteration within $6 \mathrm{~h}$ after the ictus was the most significant predictor of favorable outcome, with an OR of $10.78(\mathrm{p}<0.025)$, irrespective of treatment modality. ${ }^{23}$

Additional theoretical benefits of coiling followed by $\mathrm{ICH}$ evacuation include shorter total anesthesia time. ${ }^{24-30}$ In our study and in others, coil embolized patients have been shown to undergo shorter durations of general anesthesia and, in some institutions, may not undergo general anesthesia at all. ${ }^{31}$

Table 5 Hospitalization costs for patients with aneurysm clipping with intracerebral hemorrhage evacuation compared with coiling followed by intracerebral hemorrhage evacuation (adjusted to \$2009)

\begin{tabular}{llll}
\hline & Clip + ICH evacuation & Coil + ICH evacuation & p Value \\
\hline Total direct cost & $72792(15773-105428)$ & $71707(25356-104783)$ & 0.929 \\
Overhead & $26605(4904-44341)$ & $25141(8274-46753)$ & 1.000 \\
ICU direct cost & $28167(6860-56255)$ & $25732(3931-43994)$ & 0.424 \\
Surgery direct cost & $6796(3927-26317)$ & $8795(2543-37552)$ & 0.633 \\
Imaging direct cost & $6754(920-36431)$ & $7871(2756-27003)$ & 0.929 \\
\hline
\end{tabular}

Values are median (range).

$\mathrm{ICH}$, intracerebral hemorrhage.
Securing a ruptured aneurysm prior to a craniotomy and ICH evacuation may also have surgical technical advantages. Coiling the aneurysm prior to surgery obviates the need for more significant exposure and brain retraction, which would be necessary for open aneurysm neck clipping. Retraction of already contused or injured brain parenchyma, due to an $\mathrm{ICH}$, can be especially deleterious, as brain retraction can elicit significant edema. ${ }^{32}$ Furthermore, brain retraction in aneurysm surgery has been shown to uncouple cerebral blood flow and metabolism leading to ischemia and infarction. ${ }^{32}$ Aneurysm coiling prior to ICH evacuation has the additional benefit of virtually eliminating the possibility of intraoperative aneurysm rupture, a profound operative complication that can lead to catastrophic outcomes, including death. In patients with SAH and ICH, Houkin et al found that $12.5 \%$ of intraoperative ruptures were due to hematoma removal and $16.7 \%$ were due to brain retraction alone. ${ }^{33}$

The strategy of coil embolization followed by surgical ICH evacuation has been reported in two other case series. In a series of nine patients with ICH and $\mathrm{SAH}$, all of whom were Hunt-Hess grade III or IV, 6/9 (67\%) had favorable outcomes after 3 months with no mortalities. ${ }^{34}$ Another series of 27 patients (primarily World Federation of Neurological Surgeons grade IV or V) reported a $21 \%$ mortality rate and $48 \%$ favorable outcome rate with a median follow-up of 12 months. ${ }^{35}$ In our study, among coiled patients, the median Hunt-Hess grade was IV, mortality rate was $30 \%$ and only $30 \%$ had a good outcome (GOS 4-5). The 3 month outcome in the coiled group in our study was worse than that reported in the literature but all of these studies were small and retrospective and outcome data were reconstructed from chart review, making direct comparison difficult. Although not significantly different, the coiled group tended to be sicker than the clipped group, with lower Glasgow Coma Scale scores, higher ICH and modified Fisher scores and more herniation at presentation. Additionally, the coiled group had higher rates of hemicraniectomy than the clipped group (70\% vs $38 \%$ ), implying more significant mass effect from the $\mathrm{ICH}$. The coiled group also had a longer time from presentation to completion of aneurysm protection and ICH evacuation than the clipped group (1146 vs $885 \mathrm{~min}$ ). Despite a worse baseline neurological status, and longer time to completion of both procedures, the coiled group had similar outcomes to the clipped group. This suggests that coiling followed by ICH evacuation may be a viable alternative to simultaneous aneurysm clipping and ICH evacuation. 
Some limitations to this study should be mentioned. Firstly, this is a very small cohort. Given the incidence of SAH and ICH, a multicenter study would be required to more thoroughly compare treatment modalities. Secondly, this is a retrospective study and there may be selection bias in determining which patients underwent clipping versus coiling, although our protocol was to attempt coiling first, if possible. Additionally, 3 month functional outcome data were reconstructed from chart review. Finally, we were unable to collect reliable information regarding the use of aminocaproic acid in the two groups, which may impact the assessment of aneurysmal rebleeding, or osmotic therapy, which may affect herniation rates. An ongoing prospective trial examining coiling versus clipping with ICH evacuation is underway at our institution and may provide further insight into treatment strategies.

In conclusion, in this small retrospective study, we found that aneurysm coiling followed by ICH evacuation allowed for faster aneurysm protection without a significant difference in complications, outcome, cost or LOS compared with simultaneous clipping and ICH evacuation. Coiling followed by ICH evacuation may be a reasonable treatment option.

Competing interests None.

Ethics approval The study was approved by Mount Sinai Hospital institutional research board.

Contributors JAF and KDLR collected all of the data, performed the statistical analysis and wrote the paper. JBB and AP assisted in data analysis, and reviewed and edited the manuscript.

Provenance and peer review Not commissioned; externally peer reviewed.

\section{REFERENCES}

1. Hauerberg J, Eskesen V, Rosenorn J. The prognostic significance of intracerebral haematoma as shown on CT scanning after aneurysmal subarachnoid haemorrhage. Br J Neurosurg 1994;8:333-9.

2. Nowak G, Schwachenwald D, Schwachenwald R, et al. Intracerebral hematomas caused by aneurysm rupture. Experience with 67 cases. Neurosurg Rev 1998;21:5-9.

3. Pasqualin A, Bazzan A, Cavazzani P, et al. Intracranial hematomas following aneurysmal rupture: experience with 309 cases. Surg Neurol 1986;25:6-17.

4. Heiskanen 0, Poranen A, Kuurne T, et al. Acute surgery for intracerebral haematomas caused by rupture of an intracranial arterial aneurysm. A prospective randomized study. Acta Neurochir (Wien) 1988:90:81-3.

5. Broderick JP, Brott TG, Duldner JE, et al. Initial and recurrent bleeding are the major causes of death following subarachnoid hemorrhage. Stroke 1994;25:1342-7.

6. Cross DT 3rd, Tirschwell DL, Clark MA, et al. Mortality rates after subarachnoid hemorrhage: variations according to hospital case volume in 18 states. J Neurosurg 2003:99:810-17.

7. Wheelock B, Weir B, Watts R, et al. Timing of surgery for intracerebral hematomas due to aneurysm rupture. J Neurosurg 1983;58:476-81.

8. Tapaninaho A, Hernesniemi J, Vapalahti M. Emergency treatment of cerebral aneurysms with large haematomas. Acta Neurochir (Wien) 1988;91:21-4.

9. Tokuda $\mathbf{Y}$, Inagawa $T$, Katoh $Y$, et al. Intracerebral hematoma in patients with ruptured cerebral aneurysms. Surg Neurol 1995:43:272-7.

10. Otani N, Takasato Y, Masaoka H, et al. Surgical outcome following decompressive craniectomy for poor-grade aneurysmal subarachnoid hemorrhage in patients with associated massive intracerebral or Sylvian hematomas. Cerebrovasc Dis 2008;26:612-17

11. Tawk RG, Pandey A, Levy E, et al. Coiling of ruptured aneurysms followed by evacuation of hematoma. World Neurosurg 2010;74:626-31.
12. Torner JC, Kassell NF, Wallace RB, et al. Preoperative prognostic factors for rebleeding and survival in aneurysm patients receiving antifibrinolytic therapy: report of the Cooperative Aneurysm Study. Neurosurgery 1981;9:506-13.

13. Hillman J, Fridriksson S, Nilsson 0 , et al. Immediate administration of tranexamic acid and reduced incidence of early rebleeding after aneurysmal subarachnoid hemorrhage: a prospective randomized study. J Neurosurg 2002;97:771-8.

14. Harrigan MR, Rajneesh KF, Ardelt AA, et al. Short-term antifibrinolytic therapy before early aneurysm treatment in subarachnoid hemorrhage: effects on rehemorrhage, cerebral ischemia, and hydrocephalus. Neurosurgery 2010;67:935-9.

15. Claassen J, Bernardini GL, Kreiter K, et al. Effect of cisternal and ventricular blood on risk of delayed cerebral ischemia after subarachnoid hemorrhage: the Fisher scale revisited. Stroke 2001;32:2012-20.

16. Frontera JA, Claassen J, Schmidt JM, et al. Prediction of symptomatic vasospasm after subarachnoid hemorrhage: the modified fisher scale. Neurosurgery 2006;59:21-7

17. Hemphill JC 3rd, Bonovich DC, Besmertis L, et al. The ICH score: a simple, reliable grading scale for intracerebral hemorrhage. Stroke 2001;32:891-7.

18. Hunt WE, Hess RM. Surgical risk as related to time of intervention in the repair of intracranial aneurysms. J Neurosurg 1968;28:14-20.

19. Teasdale G, Jennett B. Assessment and prognosis of coma after head injury. Acta Neurochir (Wien) 1976;34:45-55.

20. Bederson JB, Connolly ES Jr, Batjer HH, et al. Guidelines for the management of aneurysmal subarachnoid hemorrhage: a statement for healthcare professionals from a special writing group of the Stroke Council, American Heart Association. Stroke 2009:40:994-1025

21. Naidech AM, Janjua N, Kreiter KT, et al. Predictors and impact of aneurysm rebleeding after subarachnoid hemorrhage. Arch Neurol 2005;62:410-16.

22. Wartenberg KE, Schmidt JM, Claassen J, et al. Impact of medical complications on outcome after subarachnoid hemorrhage. Crit Care Med 2006;34:617-23.

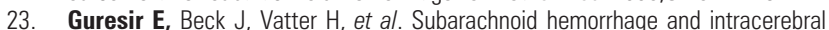
hematoma: incidence, prognostic factors, and outcome. Neurosurgery 2008;63:1088-93.

24. Badrinath SS, Bhaskaran S, Sundararaj I, et al. Mortality and morbidity associated with ophthalmic surgery. Ophthalmic Surg Lasers 1995;26:535-41.

25. Clarke-Pearson DL, Dodge RK, Synan I, et al. Venous thromboembolism prophylaxis: patients at high risk to fail intermittent pneumatic compression. Obste Gynecol 2003;101:157-63.

26. Fisher BW, Majumdar SR, McAlister FA. Predicting pulmonary complications after nonthoracic surgery: a systematic review of blinded studies. Am J Med 2002;112:219-25.

27. Korinek AM. Risk factors for neurosurgical site infections after craniotomy: a prospective multicenter study of 2944 patients. The French Study Group of Neurosurgical Infections, the SEHP, and the C-CLIN Paris-Nord. Service Epidemiologie Hygiene et Prevention. Neurosurgery 1997;41:1073-9.

28. Rebollo MH, Bernal JM, Llorca J, et al. Nosocomial infections in patients having cardiovascular operations: a multivariate analysis of risk factors. J Thorac Cardiovasc Surg 1996;112:908-13

29. Schaepkens Van Riempst JT, Van Hee RH, Weyler JJ. Deep venous thrombosis after laparoscopic cholecystectomy and prevention with nadroparin. Surg Endosc 2002;16:184-7.

30. Sinclair DR, Chung F, Mezei G. Can postoperative nausea and vomiting be predicted? Anesthesiology 1999;91:109-18.

31. Lai YC, Manninen PH. Anesthesia for cerebral aneurysms: a comparison between interventional neuroradiology and surgery. Can J Anaesth 2001:48:391-5.

32. Yundt KD, Grubb RL Jr, Diringer MN, et al. Cerebral hemodynamic and metabolic changes caused by brain retraction after aneurysmal subarachnoid hemorrhage. Neurosurgery 1997; 40:442-50.

33. Houkin K, Kuroda S, Takahashi A, et al. Intra-operative premature rupture of the cerebral aneurysms. Analysis of the causes and management. Acta Neurochir (Wien) 1999:141:1255-63.

34. Jeong JH, Koh JS, Kim EJ. A less invasive approach for ruptured aneurysm with intracranial hematoma: coil embolization followed by clot evacuation. Korean J Radio 2007:8:2-8.

35. Niemann DB, Wills AD, Maartens NF, et al. Treatment of intracerebral hematomas caused by aneurysm rupture: coil placement followed by clot evacuation. J Neurosurg 2003:99:843-7. 